\title{
Medical Use of Povidone Iodine Against Covid-19. Why Not?
}

\author{
Luis Mendoza* \\ Medical Director, Medical and Scientific Services, IQVIA, Czech Republic
}

*Corresponding author: Luis Mendoza, Senior Medical Director, Medical and Scientific Services, IQVIA, Czech Republic, Email: Luis.mendoza@iqvia.com

Received Date: May 06, 2020

Published Date: May 29, 2020

\section{Opinion}

Povidone-iodine (PVP-I) is an old antiseptic used in practice of medicine as a surgical scrub; for pre- and post-operative skin cleansing; for the treatment and prevention of infections in wounds, decubitus ulcers, cuts, and burns; in gynecology for vaginitis associated with candidal, trichomonal or mixed infections. For these purposes, PVP-I has been formulated at concentrations of 7.5$10.0 \%$ in solution, nasal and throat spray, surgical scrub, ointment, swab dosage forms, eye drop, and vaginal suppositories. The safety profile of PVP-I at such concentrations is well established and many available products in the market are over the counter. The most well-known brand for PVP-I is BETADINE.

PVP-I has been reported as a broad-spectrum microbicide with potency to inactivate bacteria, fungi, protozoans, and several viruses. After searching the medical database "PubMed" and entering the keywords: povidone-iodine and virus, I have found that there 101 scientific publications connect with PVP-I against viruses. The first publication about the efficacy of PVP-I was reported in 1975 where the PVP-I can reduce the titers of herpesvirus type 2 by $92 \%$ [1].

Recent in vitro studies have demonstrated virucidal activity of PVP-I against a wide range of enveloped and non-enveloped viruses and rapid virucidal activity against the Ebola virus, MERS-CoV, and European reference enveloped virus [modified vaccinia virus Ankara (MVA)\} [2,3]. The first evidence of the virucidal activity of PVP-I against SARS coronavirus was published in 2006 [4]. German researchers demonstrated the virucidal activity of PVP-I as skin cleanser against the Ebola virus and as gargle/mouthwash against MERS-CoV and MVA. [2]. Thanks to the funding from Mundipharma Research GmbH \& Co, German researchers published the rapid inactivation of SARS-CoV, MERS-CoV, influenza virus A (H1N1), and rotavirus after 15 seconds of exposure [5]. Mundipharma Research have developed several PVP-I based products and a gargle/ mouthwash.

In contrast to other antiseptic agents, PVP-I oral care products do not lead to any irritation or damage to the oral mucosa, even with prolonged use [5]. Respiratory Syndrome (MERS) coronavirus or endemic human coronaviruses, which is killing thousands of human beings currently, can persist on inanimate surfaces like metal, glass, or plastic for up to 9 days where the PVP-I (0.23-7.5\%) readily inactivated coronavirus infectivity by approximately 4 $\log 10$ or more [6]. In a recent paper released on 27 March 2020 in the journal Lancet Infectious Disease, the French researchers confirmed the high viral loads in upper respiratory tract samples are suggestive of the potentially high risk of transmissibility during the very first days of symptoms [7]. This finding is in line with data reported by Zou and colleagues beginning of this year, who analyzed viral load in the upper respiratory tract in relation to day of onset of symptoms in 17 symptomatic patients in whom higher viral loads were detected soon after symptom onset [8].This observation suggests that the virus shedding pattern of patients infected with COVID-19 have a high viral load in the upper respiratory tract at the disease onset.

So far, here is no vaccination or any specific antiviral treatment available for COVID-19. The COVID-19 pandemic is controlled with can, however, be quickly and effectively controlled with preventive strategies based upon early accurate viral diagnosis and adequate hygiene practices to decrease the risk of transmission.

Considering the proven in vitro efficacy of PVP-I, I am convinced that it would be an effective method of preventing the growth and spread out of the viruses-containing airborne/droplet from the nose and mouth of an infected individual. It is also possible that 
a reduction of COVID-19 viral load at nose, nasopharynx and oropharynx may help to the individual to present less severe disease and reduce the number of deaths. The destruction of the COVID-19 viral load at the upper respiratory tract will reduce of infectivity of infected individual in the community and consequently help to eradicate the COVID-19 pandemic and all terrible consequences.

On the market, several PVP-I formulations and presentations are available that differ in their composition and indications. Therefore, my suggestion for achieving an efficient anti-COVID-19 effect will be to recommend the frequent daily use of the PVP- based mouthwash for gargles and mouth rinse, combined with the PVP-I based nasal rinse or nasal spray or the application of the PVP-I based ointment to prevent the COVID-19 infection through nasal foramens. The use of the PVP-I products against COVID-19 is empiric and it may be used at least 4 times a day for health worker professionals or other professional exposure to large number of potential or COVID-19 positive individuals. For COVID-19 negative patients and for whose follow careful the recommendation of social isolation, the use of 1 or 2 times a day of PVP-I products for disinfection of nasopharynx and oropharynx might be enough. The length of PVP-I products as a prophylactic should till the risk of infectivity by COVID-19 is considered controlled by local authorities. Every country has different PVP-I products in the market, therefore the physician should look at of the available products a recommended the use for the viral cleaning and protection. The mouthwash shouldn't be swollen. The PVP-I used in humans is innocuous, but the physician should be aware some allergic (hypersensitivity) cases have been reported. In case of allergic reactions, the PVP-I should be discontinued immediately. Several cases of thyroid dysfunction induced by transcutaneous absorption of povidoneiodine have been reported. Therefore, the monitoring of the thyroid function is recommended in patients with thyroid diseases. I must also highlight here that besides the use of the PVP-I products, it is imperative to continue following the recommendations about the necessary protective measures advised to the public by WHO.

Finally, even though the chemical composition is known, the reliability of virucidal activity of the marketed product cannot be predicted. Therefore, it is essential to test the PVP-I products in clinical trials to confirm the efficacy of them against COVID-19 primary infestation at the upper respiratory tract. In that regard, there are 3 ongoing COVID-19 clinical trials to demonstrate the prophylactic and therapeutic benefit of PVP-I as nasal alone and combined with gargle applications in USA. Meanwhile, not having therapeutic alternative against COVID-19, to enhance the preventive strategies, avoid movement and self-isolation to avoid the contagious or infectivity of the virus at the upper respiratory tract, the advice of health care and system to use of low-cost PVP-I products would be key to minimize the tragedy of the current COVID-19 pandemic. Why not!

\section{Acknowledgement}

No.

\section{Conflict of Interest}

No conflict of interest.

\section{References}

1. Amsley MS, Metcalf S (1975) Effect of povidone-iodine on herpesvirus type 2, in vitro. Obstet Gynecol. 46(5): 528-529.

2. Eggers M, Eickmann M, Kowalski K, Zorn J, Reimer K (2015) Povidoneiodine hand wash and hand rub products demonstrated excellent in vitro virucidal efficacy against Ebola virus and modified vaccinia virus Ankara, the new European test virus for enveloped viruses. BMC Infect Dis 15: 375 .

3. Eggers M, Eickmann M, Zorn J (2015) Rapid and effective virucidal activity of povidone-iodine products against Middle East respiratory syndrome coronavirus (MERS-CoV) and modified vaccinia virus ankara (MVA) Infect Dis Ther 4: 491-501.

4. Kariwa H, Fujii N, Takashima I (2006) Inactivation of SARS coronavirus by means of povidone-iodine, physical conditions, and chemical reagents. Dermatology 212(Suppl 1): 119-123.

5. Rahn R, Adamietz IA, Boettcher HD (1997) Povidone-iodine to prevent mucositis in patients during antineoplastic radiochemotherapy. Dermatology 195(Suppl 2): 57-61.

6. Kampf G, Todt D, Pfaendr S, Steinmann E (2020) Persistence of coronavirus on inanimate surfaces and their inactivation with biocidal agents. J Hosp Infect 104( 3): 246-251.

7. Lescure FX, Bouadma L, Nguyen D (2020) Clinical and virological data of the first cases of COVID-19 in Europe a case series: The Lancet Infectious Diseases.

8. L Zou, F Ruan, M Huang (2020) SARS-CoV-2 viral load in upper respiratory specimens of infected patients. J Med 382(12): 1177-1179. 